\title{
LOS MÉTODOS Y PROCESOS MULTICRITERIO PARA LA EVALUACIÓN
}

\author{
ALBERTO GRAJALES-QUINTERO ${ }^{1}$, EDGAR D. SERRANO-
} MOYA ${ }^{2}$, CHRISTINE M. HAHN VON-H. ${ }^{3}$

Recibido el 7 de diciembre de 2012 y aprobado el 14 de marzo de 2013

\begin{abstract}
RESUMEN
El presente artículo tiene por objeto evidenciar las bondades que el método multicriterio otorga en las evaluaciones científicas que sean consistentes con un marco de racionalidad. Inicia haciendo referencia al método científico a través del cual el hombre trata de entender el mundo, construyendo uno artificial desde la ciencia. A continuación se reflexiona sobre el valor agregado que pueden proporcionar los métodos cualitativos al entregar una visión diferente del mundo, al tomar en consideración variables que no pueden ser expresadas cuantitativamente. Para finalizar se expone el método multicriterio como una herramienta útil para determinar el impacto de acciones a desarrollo sobre la sostenibilidad al incorporar los conflictos que existen entre objetivos económicos, ambientales y sociales, y entre distintos niveles de decisión en las evaluaciones científicas.
\end{abstract}

PALABRAS

CLAVE

Método científico, métodos cualitativos, método multicriterio.

\section{MULTICRITERIA METHODS AND PROCESSES FOR ASSESSMENT}

\section{ABSTRACT}

This article aims to make clear the benefits the multicriteriamethod gives to scientific assessment which are consistent with a rationality framework. It begins by referring to the scientific method, through which humankind tries to understand the world building an artificial world from science. Afterwards a reflection on the added value qualitative methods as a methodology can give, is presented since they allow a broader worldview by taking into account variables that cannot be expressed quantitatively. Finally, a multicriteria-method is presented as a useful tool to determine the impact of actions on sustainability, while incorporating the existing conflicts between economic, environmental and social objectives, and between different levels of decision making in scientific assessment.

\section{KEY WORDS}

Scientific method, qualitative methods, multicriteria method. 


\section{EL MÉTODO CIENTÍFICO}

El método científico es un procedimiento complejo que permite describir la realidad, diseñar escenarios de acontecimientos y señalar caminos posibles para establecer algún control sobre situaciones futuras (Cordón, 2008; Moselhi \& Roofigari-Esfahan, 2012). Para ello es necesario que se articule la teoría y su verificación con la realidad empírica, acorde con una serie de requisitos, utilizando procedimientos y técnicas establecidas y muchos de ellos probados de diversa índole. Lejos del azar, del capricho o de la simple opinión, el método ha de estructurarse en una serie de reglas que permitan un uso general e independiente de la capacidad de los sujetos que lo utilizan (Vizcaíno, Picuasi y Quiroz, 2011).

Bunge (1985) manifiesta cómo el hombre trata de entender el mundo sobre la base de su inteligencia imperfecta pero perfectible; el hombre intenta enseñorearse de él para hacerlo más confortable. En este proceso se construye un mundo artificial compuesto de un cuerpo de ideas que se denomina 'ciencia', caracterizada como conocimiento racional, sistemático, exacto, verificable y por consiguiente falible. Por medio de la investigación científica el hombre ha alcanzado una reconstrucción conceptual del mundo que es cada vez más amplia, profunda y exacta, de ahí que no sea un agregado de informaciones inconexas sino un sistema de ideas conectadas lógicamente entre sí, es decir, sistemáticas.

Así, a través de las diferentes metodologías seguidas en cada área de estudio, examinamos los procedimientos por medio de los cuales se prueben o desechen teorías y las razones por las que se prefieran unas a otras (Gómez, 2004).

En los diversos procesos metodológicos, el más sencillo y frecuente es el que va de la observación a la elaboración de hipótesis y a la deducción de resultados, los que se contrastan en la realidad-generalmente a través de un experimento o simulación con modelos formales- y se interpretan e integran en un sistema teórico (Rodríguez \& Valldeoriola, 2009). Una vez terminado un ciclo se inicia otro, tomando en cuenta los logros alcanzados, por lo que el desarrollo de la ciencia es acumulable y algunas veces progresivo -con independencia de la forma evolutiva y revolucionaria en que lo haga-.

En ocasiones se obtienen datos -por observación- que se analizan e interpretan teóricamente -generalmente sobre bases estadísticas, como análisis correlacionales- sin que sea precisa su contrastación empírica posterior (Gómez, 2004). Sin embargo para hacer 'ciencia positiva', en tanto los hechos reales son verificados por la experiencia, deben integrarse el nivel teórico con el empírico, así siempre tiene que haber una hipótesis y datos de la experiencia (Blaug, 1993).

La 'ciencia' se despliega como un arreglo interdependiente de ideas, caracterizado por cierto conjunto básico -pero refutablede hipótesis peculiares, que procura adecuarse a una clase de 
hechos; es una teoría (Carrillo, 1994). Asimismo, todo capítulo de una ciencia especial contiene teorías o sistemas de ideas que están relacionadas lógicamente entre sí, ordenadas mediante la relación "implícita". Esta conexión entre las ideas puede calificarse de orgánica en el sentido de que la sustitución de cualquiera de las hipótesis básicas produce un cambio radical en la teoría o grupo de teorías (Bunge, 1985).

Según Lakatos (2007) un programa de investigación no es más que un conjunto de reglas metodológicas que nos definen cuáles son los senderos a seguir y cuáles los problemas a evitar para la elaboración de nuevas teorías. Algunas nos dicen las rutas de investigación que deben ser evitadas (heurística negativa), y otras los caminos que deben seguirse (heurística positiva). De esta manera, la heurística suministra el marco conceptual del crecimiento científico en términos de cambios progresivos y regresivos de problemáticas para series de teorías científicas (Furio, 2005).

De lo anterior se sigue que el fundamento de una teoría dada no es un conjunto de hechos sino, más bien, un conjunto de principios, o hipótesis de cierto grado de generalidad y, por consiguiente, de cierta fertilidad lógica. A la vez, las conclusiones (o proposiciones) pueden extraerse de los principios, sea en la forma natural o con la ayuda de técnicas especiales que involucran operaciones matemáticas, así el carácter formal del conocimiento científico -esto es, el hecho de que es fundado, ordenado y coherente- es lo que le otorga una condición de racionabilidad que permite, en últimas, que el progreso científico se efectúe no solo por la acumulación gradual de resultados, sino también por revoluciones (Moselhi \& Roofigari-Esfahan, 2012).

\section{LOS MÉTODOS CUALITATIVOS}

En la evaluación de proyectos, en diversos programas de investigación social, política económica, entre otros, no se agota toda la información disponible sobre problemas o necesidades que estos pretenden solucionar; la recopilación de información económica es una condición necesaria en diversos proyectos pero no suficiente cuando se integra la descripción de variables sociales (Hernández, Fernández \& Baptista, 2006). Una de las características principales de las metodologías multicriterio es la diversidad de factores que se logran integrar en el proceso de evaluación (Arancibia et al., 2005). Existe una gran cantidad de factores que no pueden ser valorados para ser incluidos en un análisis Costo-Beneficio o que son excluidos en los análisis Costo-Eficiencia (Contreras \& Pacheco, 2007).

Entendiendo que una variable es una característica de la realidad que puede ser determinada por observación y puede tomar diferentes valores de una unidad de observación a otra, en general se establece que dependiendo de la naturaleza de la investigación y del tipo de información que trate, las variables 
pueden agruparse en dos tipos, cuantitativas y cualitativas ${ }^{5}$ (Rodríguez \& Valldeoriola, 2009).

Así, las decisiones deben tomarse considerando no solo aspectos económicos, ya que no todos los factores que se modifican con una intervención son cuantitativamente expresables de modo tradicional o monetario. Es difícil cuantificar el impacto ambiental y las consecuencias al realizar un proyecto, los cuales poseen gran importancia en programas de provisión de bienes públicos. Consecuentemente, el diseño y la realización de estas, son de capital importancia, debiendo contar a lo largo de todo el proceso con factores como la percepción, intuición, experiencia y otros, que son cualidades no valorables de un problema y que no pueden excluirse al abordarlo. Por otra parte, un programa o proyecto con frecuencia afecta no solo a las variables que se pretenden intervenir, sino que trae consecuencias adicionales, muchas de ellos no deseadas o inesperadas, consecuencia de la complejidad de las interacciones sociales y sus diversas facetas (Contreras \& Pacheco, 2007).

Un ejemplo hipotético: un municipio desea reducir la delincuencia en una zona; para esto evalúa dos alternativas, construir una cancha de fútbol o una plaza con áreas verdes. Decide por una de ellas en función de una evaluación económica -la menos costosa-. La decisión adoptada por el municipio sería la "correcta" si el camino obligatorio fuera el bajo costo económico (Contreras \& Pacheco, 2007). Sin embargo, la toma de decisión no recoge toda la información disponible para la toma de decisiones. Hay un grupo de criterios cualitativos que se pueden agregar al análisis antes expuesto, supongamos que existe: aficionados que están polarizados por un equipo de fútbol en la zona del proyecto, o que hay grupos etarios que se definen claramente dentro de las personas involucradas en situaciones de actividades ilícitas, nivel de drogadicción en la zona, etc.

Estos criterios hacen la diferencia al momento de evaluar la alternativa del proyecto, así si las preferencias por equipos de fútbol es baja, no sería conveniente construir la cancha de fútbol, pues en un ambiente de alta delincuencia podría conducir a mayores niveles de violencia. Por el contrario, si el nivel de drogadicción juvenil es alto, es preferible construir la cancha en vez de la plaza (Contreras \& Pacheco, 2007).

La obviedad del ejemplo indica que sería necesario incluir los aspectos antes descritos para mejorar la toma de decisiones, pero el gran número de factores y naturaleza de algunos de ellos hace difícil su inclusión en las formas tradicionales de evaluación de proyectos, sin embargo las técnicas de "Evaluación Multicriterio" permitirían trabajar con varios criterios a la vez e identificar la importancia relativa de cada uno para evaluar entre distintas alternativas de proyecto, independiente de la naturaleza de los factores que están implícitos en una situación específica (Pacheco \& Contreras, 2008). 
En el mundo actual podrá asumirse racionalmente que para cualquier problema de toma de decisiones existe una solución óptima precisa y que es posible encontrarla razonando respecto al problema y modelándolo adecuadamente (Contreras, 2004).

La "Evaluación de Proyectos" -con enfoque de eficienciasupone la capacidad de identificar, medir y valorar, todos los costos y beneficios involucrados, cuestión que en la práctica no siempre es posible. Así, los beneficios y costos que se deben identificar, medir y valorar, son aquellos que resulten relevantes desde el punto de vista del inversionista que desea llevar a cabo el proyecto (Conteras \& Pacheco, 2007). Especialmente se distinguen dos puntos de vista, uno es el del negociante privado y/o oficial, y otro es el de todos los agentes económicos que conforman la comunidad nacional (Calderón, Ríos \& Ceccarini, 2008). Con frecuencia este último análisis se aplica a políticas y programas que presentan externalidades (aspectos que están por fuera de las valoraciones de mercado) como, por ejemplo, el mejoramiento en la calidad ambiental (RodríguezBecerra \& Espinoza, 2002). En este escenario, es posible buscar consensos en torno a la evaluación de proyectos, incorporar las variables no cuantificables o factores subjetivos que pesan en las decisiones de los agentes económicos, e incorporar procesos de toma de decisiones que son crecientemente grupales (Roura \& Cepeda, 1999; Gómez et al., 2009).

\section{Momentos del proceso de investigación}

El interés por la cuantificación en las ciencias sociales, en general, y en la geografía, en particular, es de larga data. C. Escolar y J. Besse (1996 citados por Pedone, 2000) introducen una recopilación de artículos que tratan sobre el uso de metodología y técnicas cualitativas y proponen:

[...] una perspectiva epistemológica desde la cual reflexionar los problemas de los métodos, así como una manera más específica de abordar la relación entre teoría, método y técnicas cualitativas en un proceso de investigación [...] la falsa oposición entre técnicas cuantitativas y cualitativas sólo sirvió para delinear fronteras rígidas y plantear la opción por lo cuantitativo de manera dilemática y no problemática (Escolar y J. Besse (1996) citado por Pedone, 2000, p 15 y 2)

Distintos autores identifican este dualismo en diferentes momentos del proceso de investigación. Brannen (1992 citado por Pedone, 2000) sostiene que las aproximaciones cualitativas permiten una visión del mundo más amplia que los enfoques cuantitativos, y Hammersley (1992 citado por Pedone, 2000) presenta una lista de dicotomías entre los métodos cuantitativos y cualitativos en general (Tabla 1). 
Como se muestra en la Tabla 1, las mediciones cuantitativas son pequeñas a partir de muestreos aleatorios de una muestra amplia, por el contrario, las entrevistas cualitativas realizan muestreos dirigidos en muestras pequeñas. Los métodos cuantitativos muestran posiciones verdaderas a partir de leyes científicas basadas en escenarios experimentales cuantitativos; mientras que los métodos cualitativos presentan perspectivas idealistas a partir de patrones culturales y buscan el conocimiento con base en escenarios naturales cualitativos (Duglio, 2007; Armada de México, 2010).

Tabla 1. Dualismos identificados entre métodos cualitativos y cuantitativos por Hammersley

\begin{tabular}{||c||c||}
\hline \multicolumn{1}{|c||}{ Métodos Cualitativos } & Métodos Cuantitativos \\
\hline \hline Datos cualitativos & Datos cuantitativos \\
Escenarios naturales & Escenarios experimentales \\
Búsqueda de conocimiento & Identificación de comportamiento \\
Rechazo a la ciencia natural & Adopción de la ciencia natural \\
Aproximaciones inductivas & Aproximaciones deductivas \\
Identificación de patrones culturales & Consecución de leyes científicas \\
Perspectiva idealista & Perspectiva realista \\
\hline \hline Entrevistas Cualitativas & Mediciones Cuantitativas \\
\hline \hline Muestra de tamaño pequeño & Muestra de tamaño amplio \\
Entrevistas extensas & Mediciones pequeñas \\
Muestreos no-aleatorios & Muestreo aleatorio \\
\hline Fuente: Winchester (1996 citado por Pedone, 2000, p. 2).
\end{tabular}

Los investigadores que hacen uso de los métodos cualitativos, influidos por los criterios de validez de los métodos cuantitativos, desarrollan procedimientos que intentan permanentemente justificar el tamaño de su muestra, su diseño y los métodos de análisis (Winchester, 1996); autores como Sayer (1984), Allen y McDowell (1989) y McDowell (2000), coinciden en que las entrevistas cualitativas son absolutamente esenciales desde el punto de vista del realismo crítico, ya que esta postura reconoce que las estructuras que se encuentran en los estudios que analizan grupos humanos son subordinadas, complejas y pueden diferir de los hechos observados y de los discursos de los cuales emergen.

Si bien los investigadores que utilizan métodos cuantitativos, han exagerado esta división y han impuesto la primacía de sus contribuciones sobre las de sus contrapartes cualitativas, no se puede negar que los analistas cualitativos también han censurado la cuantificación como inherentemente insensible y no imaginativa. Se puntualiza, además, que en numerosos estudios la metodología cualitativa cae en algunas de las limitaciones ya observadas en los métodos cuantitativos, al suponer la existencia de formas únicas de abordar la realidad y proponer para todo tipo de investigación un conjunto de pasos prefijados del tipo cualitativo (Pedone, 2000).

Pedone (2000) no comparte la dualidad existente entre los métodos cuantitativos y cualitativos, por el contrario, respalda 
una complementariedad, puesto que la contrastación y verificación para probar la validez de la investigación debe ser propuesta por el investigador, en cada caso específico, sin estar reservada a lo cuantitativo o lo cualitativo (Linkov et al., 2006).

Combinar métodos en un mismo proyecto de investigación puede ser ventajoso por diversas razones (Philip, 1998), i.e., el uso de más de una técnica en la recolección de pruebas ayuda a minimizar los riesgos de obtener postulados erróneos. Además, se puede llevar adelante la recolección del material y luego generar datos cuantitativos como cualitativos. Los hallazgos cuantitativos permiten obtener conclusiones generales a partir de premisas particulares y los cualitativos ofrecen la posibilidad de recoger información a partir de los hechos y del ámbito en que se presentan éstos.

La práctica investigativa implica permanente toma de posiciones y decisiones por parte de los investigadores, así las técnicas, los métodos y la teoría, se constituyen en cada proceso de investigación y de acuerdo con las particularidades del objeto de estudio (Zerguini et al., 2011). Por lo anterior, las técnicas se encuentran subordinadas a la teoría en un proceso de construcción y sujetas a los procesos de reformulación de la problemática de investigación, esto permite redefinir la selección de otras técnicas para construir otros datos y enriquecer la investigación (Escolar \& Besse, 1996 citados por Pedone, 2000; Linkov et al., 2006).

Philo (1998) reconsidera la distinción entre "cantidad y calidad", y aboga por la interacción entre métodos cualitativos y cuantitativos, de tal manera que sea necesario propiciar un debate profundo de ideas, puesto que hay cuestiones éticas y conceptuales que todavía no se han resuelto y a las que es necesario responder, como el compromiso del investigador con la sociedad y sus posibles contribuciones para resolver los problemas que plantea en cada contexto.

Los métodos cualitativos asocian su surgimiento al fracaso de los enfoques cientificistas, vinculados al neopositivismo, para analizar el significado del mundo social, y por otra parte, al uso excesivo de los datos económicos y la clasificación cuantitativa que ha ignorado las múltiples aristas de la experiencia humana, y defendido la subjetividad de los sujetos y sus interacciones como objeto de estudio. Con todo ello, los datos no cuantificables, basados particularmente en experiencias y actitudes, pueden dar una visión "holística" y derivar conocimientos y explicaciones causales (Pedone, 2000).

Es así como trabajos cualitativos de evaluación multicriterio, en los cuales se involucran por ejemplo problemas ambientales, sociales y de uso de recursos en general, y donde se combinan dimensiones económicas, sociales y ecológicas, son trabajos que intentan en su integralidad minimizar las consecuencias de enfoques menos amplios en la investigación de los grupos humanos y sus interacciones (León, 2006; Astier, Masera \& Galván-Miyoshi, 2008). 
Siguiendo a Munda, Nijkamp y Rietveld (1995), es necesario utilizar modelos que ofrezcan una representación comprensible y operacional del mundo real. Para ello es recomendable el uso de modelos de evaluación multicriterio cuyo impacto permite la interacción entre juicios cuantitativos y cualitativos, que permiten describir una colección de conceptos, métodos y técnicas que ayudan a los individuos o grupos a tomar decisiones cuando se involucran diferentes puntos de vista en conflicto y múltiples agentes interesados (Salo \& Hamalainen, 2010).

Es condición para un problema de decisión multicriterio la presencia de más de un criterio, y que éstos se encuentren en conflicto. Es decir, un problema puede considerarse como problema multicriterio si y sólo si existen al menos dos criterios en conflicto y existen al menos dos alternativas de solución, para definir los atributos que se utilizan para describir alternativas que pueden ser cuantitativas (objetivas) 0 cualitativas (subjetivas) y una meta u objetivo (González \& Garza, 2003).

\section{LA EVALUACIÓN MULTICRITERIO}

La opinión autocrática en la toma de decisión puede tornarse insuficiente cuando se analizan problemas complejos, sobre todo aquellos problemas en donde la solución puede afectar a muchas otras personas (Munda, 2004; Mendoza \& Martins, 2006; Dou, Zhu \& Simon, 2012). Debido a lo anterior, se debe propender por generar discusiones e intercambio de ideas y opiniones entre expertos (Tabla 2), quienes por su experiencia y conocimiento pueden ayudar a estructurar el problema y a evaluar las posibles soluciones (Hernández, Fernández \& Baptista, 2006; Dalalah, Hayajneh \& Batieha, 2011). 
Tabla 2. Modelo para la toma de decisión

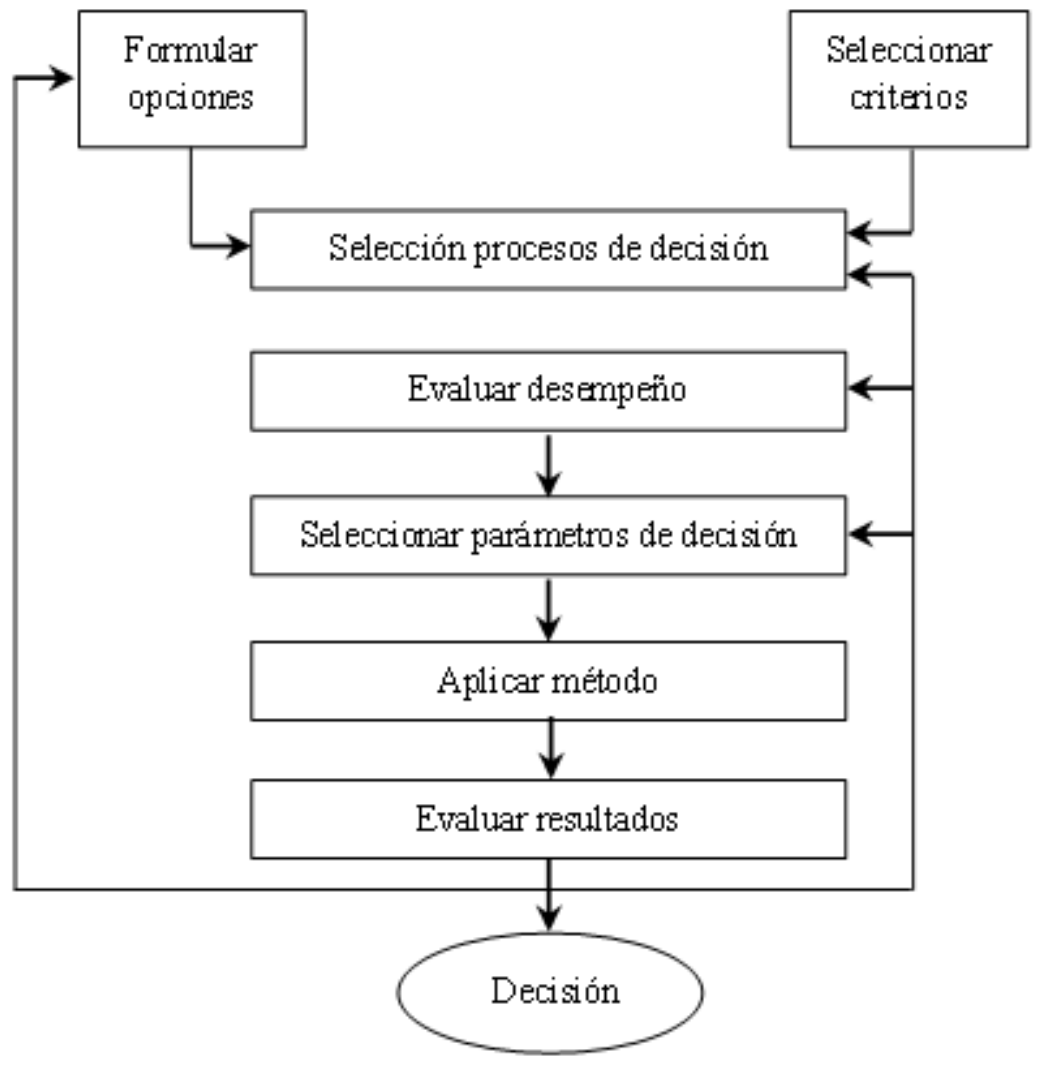

En estos casos generalmente se presentan diversos objetivos o criterios que simultáneamente deben incorporarse, los cuales requieren de la aplicación de una Metodología como la propuesta que implica la selección entre un conjunto de alternativas factibles, la optimización de varias alternativas de objetivo, y procedimientos de evaluación racionales y consistentes, que se utilizan para tomar decisiones frente a problemas que contienen aspectos intangibles a evaluar (Tabla 3) (Ortegón, Pacheco \& Roura, 2005; Dooley et al., 2009).

\section{El proceso de decisión}

El proceso de decisión requiere de la comparación entre las alternativas sobre las que se puede optar frente a cierta disyuntiva presente (Wen-Hsiang, Chang-tzu \& Chin-tsai, 2008). En primer lugar se hace necesario separar un problema de decisión en los "elementos" que lo componen para la posterior comparación entre ellos, de esta manera la toma de decisión implica realizar mediciones que permitan aplicar los criterios de comparación para establecer preferencias entre ellos (jerarquías) (Font, 2000). Si se desea tener una herramienta eficaz y eficiente que apoye las decisiones, es de importancia contar con la mayor cantidad de elementos de análisis junto al proceso más adecuado para ello (Arancibia et al., 2005; Montibeller \& Franco, 2010). 
A modo de ilustración podríamos considerar la situación que vive el sector agrícola y pecuario colombiano, la información existente sobre los diferentes sectores que componen este importante sector está desactualizada en sus diferentes componentes lo que imposibilita por ejemplo una cuantificación de la producción. En Colombia el campo es fuente de empleo, abastecedor de alimentos y de materia prima para la agroindustria, es generador de divisas, pero el ingreso de los pobladores del campo es, en general, precario, por lo que se presentan elevados niveles de pobreza y problemas sociales relacionados con la dinámica productiva.

En Colombia es necesario identificar y analizar las causas de los problemas que se presentan en el sector agrario con el fin de evaluar posibles soluciones, todo con el fin de elaborar políticas efectivas en el sector (Stirling, 2006). En este sentido el método multicriterio no considera soluciones óptimas, pero permite desde su estructura analítica en función de las preferencias de los agentes seleccionar la(s) mejore(s) alternativa(s) de solución de problemas (Toskano, 2005).

La forma de resolver un problema típico es utilizando un conjunto de teorías, métodos, modelos y herramientas de apoyo a la toma de decisiones, aplicable a una amplia gama de problemas; se hace necesaria una metodología que logre combinar las distintas dimensiones, objetivos, actores y escalas que se hallan envueltos en el proceso sin sacrificar la calidad, confiabilidad y consenso en los resultados (Gómez-Limón \& Berbel, 2000; Hajkowicz, 2007; Hajkowicz \& Higgins, 2008; Simões-Gomes et al., 2008).

Una de las posibilidades que las metodologías multicriterio aportan, es la diversidad de factores que se logran integrar en el proceso de evaluación. Su particularidad está en la forma de transformar las mediciones y percepciones en una escala única para comparar los elementos y establecer órdenes de prioridad que permitan agregar los efectos de un proyecto en una métrica común (Contreras \& Pacheco, 2007).

Por ejemplo, el estado de deterioro global y de escasez de recursos al que nuestra forma de vida ha conducido al planeta, hace necesario conciliar lo económico, social y cultural con lo ambiental, tratando de ajustar el estado de los sistemas ecológicos y sus aspectos de desarrollo. Sin embargo, las iniciativas de desarrollo que consideran las múltiples interrelaciones entre sociedad y su medio ambiente natural, no son aplicadas correctamente y desconocen el papel que desempeña la naturaleza en la economía como soporte vital de ésta.

Dado el desconocimiento de los principios y aplicaciones de la sostenibilidad ambiental, social y económica, procesos irreversibles se desencadenan sobre los ecosistemas. Así pues, las prácticas de conservación y preservación son urgentes y debe reconocerse al manejo sostenible en la normatividad ambiental (Pearce \& Turner, 1995 citado por Uribe, 2001). 
Al plantear la sostenibilidad se alude al intercambio de acciones hechas con los recursos naturales y las consecuencias hacia las generaciones futuras (Garmendia \& Gamboa, 2012). La sostenibilidad de los recursos naturales como capital natural y la tecnología desarrollada por el hombre son un concepto crítico ya que, según los factores de producción en la economía clásica, es posible sustituir al capital natural, mientras que la economía ecológica lo asume como poco conveniente (Munda, 1993).

La importancia de las técnicas de evaluación y valoración de los recursos naturales se expresa en su capacidad para convertirse en una herramienta de decisión sobre el uso sostenible de los mismos, y en que permiten emitir juicios al seleccionar estrategias de manejo y conservación (Funtowicz \& Ravetz, 1993). El bienestar es una variable multidimensional que incluye ingresos, crecimiento, calidad medioambiental, equidad, oferta de bienes públicos, entre otras (Vargas, 2005), por esta razón la toma de decisiones multicriterio da al 'decisor' herramientas que permiten avanzar en la solución de problemas en los cuales los puntos de vista son diversos y deben ser evaluados, no necesariamente como soluciones óptimas, sino como la decisión más flexible que se asume en oposición a las soluciones tomadas con las técnicas clásicas.

Métodos multicriterio. La metodología descompone un problema complejo en partes más simples permitiendo que el agente 'decisor' pueda estructurar un problema con múltiples criterios en forma visual, mediante la construcción de un modelo jerárquico que básicamente contiene tres niveles: meta $u$ objetivo, criterios y alternativas, jugando un papel vital como herramienta de planeación (Nijkamp et al., 1990 citados por Uribe, 2001; Munda, 2004; Chen et al., 2012).

Adicionalmente posee ciertas ventajas al ser comparada con las herramientas de decisión unidimensionales en la medida en que hace posible considerar un número amplio de datos, relaciones, criterios y propósitos, los cuales se presentan dentro de un problema de decisión dado en el mundo real que se estudia según un modelo multidimensional (Funtowicz et al., 1998 citados por Uribe, 2001).

La evaluación multicriterio (Munda, 1993), (i) considera factores de tipo cualitativo y cuantitativo; y (ii) considera la pluralidad de percepciones de los actores involucrados en el problema de decisión, la cual debe ser participativa para tomar decisiones y trazar alternativas para la solución de conflictos (Romero, 1997 citado por Uribe, 2001; Chen et al., 2012).

\section{Componentes de una Evaluación Multicriterio}

De acuerdo con Munda (1993) y Munda, Nijkamp y Rietveld (1995), las etapas más importantes de una evaluación multicriterio son (Tabla 3): 
Tabla 3. Componentes de una Evaluación Multicriterio

\begin{tabular}{|c|c|}
\hline Definición y estructuración del problema & $\begin{array}{l}\text { Es definido por el escenario de evaluación, } \\
\text { la disponibilidad de información y los } \\
\text { posibles conflictos entre diversos intereses } \\
\text { de cada actor involucrado. Estos intereses, } \\
\text { generalmente, se asocian al problema según } \\
\text { dimensiones múltiples de evaluación que } \\
\text { hacen necesario un tratamiento multicriterial } \\
\text { y discreto. }\end{array}$ \\
\hline Descripción de alternativas potenciales & $\begin{array}{l}\text { Considera las posibles situaciones o } \\
\text { escenarios de evaluación, es decir, son los } \\
\text { elementos sobre los cuales se decide. La } \\
\text { definición de las alternativas puede } \\
\text { realizarse tanto en una etapa previa a la } \\
\text { evaluación multicriterio como en su mismo } \\
\text { desarrollo. Generalmente el número de } \\
\text { alternativas es finito (métodos discretos) } \\
\text { pero pueden ser infinitas posibilidades de } \\
\text { elección (métodos continuos). }\end{array}$ \\
\hline $\begin{array}{l}\text { Elección de conjuntos de criterios de } \\
\text { evaluación }\end{array}$ & $\begin{array}{l}\text { Deberá contarse con abundantes conjuntos } \\
\text { de criterios de evaluación, para que el } \\
\text { problema sea mejor interpretado. Un grupo } \\
\text { de criterios debe cumplir con dos cualidades: } \\
\text { ser legible -número de criterios suficiente } \\
\text { para soportar un procedimiento de } \\
\text { agregación- y ser operativo -abarcar los } \\
\text { múltiples intereses de todos los actores } \\
\text { claves-. Los criterios deben poseer cierto } \\
\text { número de propiedades técnicas en aras de } \\
\text { su consistencia y transparencia. }\end{array}$ \\
\hline $\begin{array}{l}\text { Identificación de un sistema de } \\
\text { preferencia para la toma de decisiones y } \\
\text { elección de un procedimiento de } \\
\text { agregación }\end{array}$ & $\begin{array}{l}\text { Asignación de peso, resaltando la } \\
\text { importancia relativa de los diferentes } \\
\text { criterios de evaluación, generalmente en } \\
\text { decisiones donde no se comprometen varios } \\
\text { actores y mediante procedimientos } \\
\text { interactivos. En la conformación de las } \\
\text { variables de evaluación se involucran tanto } \\
\text { los actores como los decisores. }\end{array}$ \\
\hline
\end{tabular}

Con base en los criterios de Contreras et al. (2008), se presenta un ejemplo de Matriz Multicriterio para la priorización de problemas:

1. Una vez definido el problema en una comunidad, se llena una matriz de priorización con el grupo o equipo de trabajo, el cual está conformado con todos los actores sociales involucrados en la problemática, los cuales aportarán en la construcción de la matriz de precisión.

2. Se disponen en una matriz los criterios seleccionados en las columnas, y los problemas identificados en las filas (Tabla 4).

3. Llenado de las celdas valorando la presencia de cada criterio en cada uno de los problemas utilizando la siguiente escala: 3 Alto, 2 Medio, 1 Bajo o 0 Nulo, para cada problema, con el criterio que se esté considerando.

4. Realizar sumatoria de puntos para cada problema dispuestos en las filas.

5. Establecer un orden de prioridad atendiendo la puntuación obtenida por cada problema según la sumatoria. Tendrá mayor prioridad el problema que obtenga mayor puntaje en la sumatoria. 
Tabla 4. Matriz Multicriterio para la priorización de problemas

\begin{tabular}{||l|l|l|l|l|l|l|l||}
\hline & Criterio 1 & Criterio 2 & Criterio 3 & Criterio 4 & $\ldots$ & Criterio "N" & Suma \\
\hline \hline Problema 1 & & & & & & & \\
\hline \hline Problema 2 & & & & & & & \\
\hline \hline$\ldots$. & & & & & & & \\
\hline Problema "N" & & & & & & & \\
\hline
\end{tabular}

Fuente: Contreras et al. (2008).

Se presenta a una asociación de productores que intenta priorizar problemas comunitarios complejos de la siguiente manera:

- En primer lugar se utiliza la escala de estimación descrita anteriormente en el numeral 3.

- Para construir la matriz, se ubican en la primera columna los problemas que se consideraron previamente durante una lluvia de ideas, tales como: Incremento en el consumo de drogas entre la población de 14 a 25 años; escasez de agua potable en el área de estudio; alto índice de diarrea entre la población infantil de 0 a 4 años; y baja calidad del servicio de transporte.

- En la primera fila se sitúan los criterios utilizados, por ejemplo: La población afectada por cada uno de los problemas; la capacidad para obtener éxito al enfrentar este problema; la importancia que reviste para la asociación comunitaria; el impacto sobre otros problemas; y el costo de postergación en la atención de este problema.

- Cuando se tienen todos los criterios, se coloca una columna para la sumatoria y una columna siguiente que define la prioridad del problema, obtenida de la sumatoria.

- Para llevar a cabo el llenado de las celdas en la matriz, los actores sociales involucrados deben llegar a un consenso sobre la valoración que se le dará a cada uno de los problemas, según cada uno de los criterios, como lo describe la Tabla 5.

- Como lo ilustra la Tabla 5, para el incremento en el consumo de drogas entre la población de 14 a 25 años, los actores sociales involucrados llegaron a un consenso de que la población afectada era baja, que la capacidad para obtener éxito al enfrentarlo era medio, que la importancia para la asociación de productores era alta, así como en el impacto sobre otros problemas la valoración fue media, y finalmente el costo para la postergación fue alta.

- De la manera descrita se realiza la votación para cada uno de los problemas y se coloca el número que corresponda a la valoración de cada uno de los criterios. 
Tabla 5. Problemas comunitarios de una asociación de productores

\begin{tabular}{||c|c|c|c|c|c|c|c||}
\hline \hline & $\begin{array}{c}\text { Población } \\
\text { afectada }\end{array}$ & $\begin{array}{c}\text { Capacidad } \\
\text { para } \\
\text { obtener } \\
\text { éxito al } \\
\text { enfrentarlo }\end{array}$ & $\begin{array}{c}\text { Importancia } \\
\text { para la } \\
\text { asociación }\end{array}$ & $\begin{array}{c}\text { Impacto } \\
\text { sobre } \\
\text { otros } \\
\text { problemas }\end{array}$ & $\begin{array}{c}\text { Costo de } \\
\text { postergación }\end{array}$ & Suma & Prioridad \\
\hline $\begin{array}{l}\text { Incremento } \\
\text { en el } \\
\text { consumo } \\
\text { de drogas } \\
\text { entre la } \\
\text { población } \\
\text { de 14 a 25 }\end{array}$ & 1 & 2 & 3 & 2 & 3 & 11 & 2 \\
$\begin{array}{l}\text { años } \\
\begin{array}{l}\text { Escasez } \\
\text { de agua } \\
\text { potable en } \\
\text { el área de } \\
\text { estudio }\end{array}\end{array}$ & 1 & 2 & 2 & 2 & 1 & 8 & 3 \\
\hline $\begin{array}{l}\text { Alto indice } \\
\text { de diarrea } \\
\text { entre la } \\
\text { población } \\
\text { infantil de } \\
\text { 0 a 4 años }\end{array}$ & 3 & 2 & 3 & 2 & 3 & 13 & 1 \\
\hline $\begin{array}{l}\text { Baja } \\
\text { calidad del } \\
\text { servicio de } \\
\text { transporte }\end{array}$ & 3 & 1 & 2 & 1 & 0 & 7 & 4 \\
\hline
\end{tabular}

Fuente: adaptado de Contreras et al. (2008).

- Finalmente, cuando se han llenado todas las celdas de la tabla según las votaciones, se realizan las sumatorias. Por ejemplo, para el primer problema la suma total de cada una de las ponderaciones que se hace para cada uno de los criterios, la sumatoria es de 11 puntos; para la escasez de agua potable en la comunidad estudiada, la valoración es de 8 puntos; para alto índice de diarrea entre la población infantil de 0 a 4 años, la valoración es de 13; y finalmente para la baja calidad del servicio de transporte la valoración es de 7 puntos.

- Por último, se considera cuál de los problemas obtuvo mayor puntaje en la sumatoria, y ese problema será la prioridad número uno, es decir, ese será el problema considerado para ser abordado a fin de dar una solución a dicha problemática. Para el ejemplo, el problema prioritario resulta ser el alto índice de diarrea entre la población infantil de 0 a 4 años, debido a que cuenta con el mayor valor en la suma de ponderaciones otorgadas.

\section{A MANERA DE CONCLUSIÓN}

Al evaluar proyectos ambientales, sociales o en programas de provisión de bienes públicos, no se agota toda la información disponible sobre problemas o necesidades que estos pretenden solucionar. Para ello se requiere un compromiso entre actores, con una perspectiva de equidad, que involucra la dimensión ecológica, económica, social y cultural, otorgado por el uso del método multicriterio (Contreras \& Pacheco, 2007). 
Los métodos multicriterio son una herramienta útil para determinar el impacto de acciones de desarrollo sobre la sostenibilidad. Estos métodos permiten incorporar los conflictos que existen entre objetivos económicos, ambientales y sociales, y entre distintos niveles de decisión (Corral \& Quintero, 2007). Así, al establecer una solución ideal, pueden detallarse puntos de referencia respecto de los cuales se puede medir el impacto de diversas alternativas potenciales (IAF, 2000 citado por Uribe, 2001). Es decir, la evaluación multicriterio permite implementar soluciones-compromiso, con sentido de equidad, consenso y participación.

Estos métodos se aplican no solo a los problemas específicos de la valoración ambiental, sino que están relacionados con toda la decisión económica de los recursos naturales. Por lo tanto, no se optimizan las decisiones con base en un solo objetivo, por el contrario, se busca un equilibrio o compromiso entre un conjunto de objetivos en conflicto (Romero, 1997 citado por Uribe, 2001); un campo de trabajo coherente que hace del problema de decisión un proceso transparente en la medida en que da cuenta cabal de todos los logros y consecuencias de las diferentes estrategias de planeación (Nijkamp et al., 1990 citados por Uribe, 2001).

La evaluación multicriterio puede ayudar a encontrar soluciones-compromiso entre los diferentes objetivos en conflicto, generalmente ecológicos y económicos.

El uso de variables difusas en evaluaciones puede ser utilizada como herramienta de decisión para el manejo de problemas medioambientales caracterizados por una alta incertidumbre y evaluaciones aproximadas.

Los procedimientos matemáticos desarrollados mediante las técnicas multicriterio pueden ser una herramienta eficiente para tratar aspectos de eficiencia, de equidad y de interacciones entre la economía y el ambiente de un problema medioambiental.

Los métodos de evaluación y decisión multicriterio comprenden la selección entre un conjunto de alternativas factibles, basado en un conjunto de criterios cualitativos y/o cuantitativos, los cuales pueden estar en conflicto, por lo que es necesario optimizar varias funciones objetivo simultáneas y contar con la participación de múltiples agentes decidores y expertos, que a partir de procedimientos de evaluación racionales y consistentes, permitan tomar decisiones frente a problemas que contienen aspectos intangibles a evaluar (Toskano, 2005).

El fundamento del método de toma de decisión multicriterio se basa en descomponer un problema complejo en partes más simples, lo cual permite que el agente 'decisor' pueda estructurar un problema con múltiples criterios en forma visual, mediante la construcción de un modelo jerárquico que básicamente contiene tres niveles: meta u objetivo, criterios y alternativas (Sainz, Álvarez \& Henríquez, 2012). Una vez 
construido el modelo se realizan comparaciones de a pares entre dichos elementos (criterios-subcriterios y alternativas) y se atribuyen valores numéricos a las preferencias señaladas por las personas, entregando una síntesis de las mismas mediante la agregación de esos juicios parciales.

Los métodos multicriterio y la Lógica difusa se han implementado con éxito en diversas áreas como la selección, evaluación, análisis de costo-beneficio, asignación, planeación y desarrollo, prioridad y ranking, toma de decisión, predicción, salud, administración, finanzas, calidad, cadena de suministro, entre otras.

\section{REFERENCIAS}

- Allen, J. \& McDowell, L. (1989). Landlords and property: social relations in the private rented sector. Extraído el 23 de febrero de 2011 de http://books.google.com.co/books?id=cZiT1utcGxM C\&printsec $=$ frontcover\&dq=Landlords+and+Propert y\%2BAllen+y+McDowell\&source=bl\&ots=HNw1fHcy

P\&sig=7wwTBM7Gnb7|1_4hcw7cjfzzC9s\&hl=es\&sa $=\mathrm{X} \& \mathrm{e}=$ YbE3UNnYOqfs0gHv54CICw\&ved $=0 C C 4 Q 6 A E w A A \# v=$ onepage $\& q=$ Landlords $\% 20$ and $\% 20$ Property \%2BAllen\%20y\%20McDowell\&f=false

- Arancibia, S., Contreras, E., Mella, S., Torres, P. \& Villablanca, I. (2005). Evaluación Multicriterio: aplicación para la formulación de proyectos de infraestructura deportiva. Santiago de Chile. Extraído el 23 de febrero de 2011 de http://www.dii.uchile.cl/ ceges/publicaciones/ceges 48.pdf

- Armada de México. (2010). Secretaría de Marina. Manual para elaborar y evaluar trabajos de investigación. Extraído el 14 de junio de 2011 de http://www.cesnav.edu.mx/pdfs/manual_de_investig acion.pdf

- Astier, M., Masera, O. R. y Galván-Miyoshi, Y. (2008). Evaluación de sustentabilidad. Un enfoque dinámico y multidimensional. Extraído el 14 de junio de 2011 de http://www.ciga.unam.mx/ciga/images/stories/public aciones/ sustentabilidad/GIRA_CS3_final.pdf

- Blaug, M. (1993). La Metodología de la Economía. 2 ed. Madrid: Alianza Editorial. 330p. ISBN: 9788420624242.

- Bunge, M. (1985). La ciencia. Su método y su filosofía. Extraído el 28 de febrero de 2011 de http://www.aristidesvara.net/pgnWeb/metodologia/ metodo_cientifico/naturaleza_metodo/bunge_libro_ aristidesvara.pdf 
- Calderón, M., Ríos, M. A. y Ceccarini, M F. (2008). Economía de la educación. Extraído el 25 de agosto de 2011 de http://bdigital.uncu.edu.ar/objetos_digitales/2420/ec onomiadelaeducacion.pdf

- Carrillo, L. R. (1994). Metodología y Administración. El método científico.: Ed. Limusa Noriega. México

- Contreras, E. (2004). Evaluación social de inversiones públicas: enfoques alternativos y su aplicabilidad para Latinoamérica. Extraído el 18 de julio de 2012 de http://www.eclac.cl/publicaciones/xml/2/20962/manu al37.pdf

- Contreras, E. y Pacheco, J. F. (2007). Evaluación Multicriterio para Programas y Proyectos Públicos. Extraído el 10 de marzo de 2011 de http://www.sistemaspublicos.cl/wp-content/ files_mf/127258066992-ceges-EC.pdf

- Contreras, F., Hanakia, K., Aramakia, T. \& Connors, S. (2008). Application of analytical hierarchy process to analyze stakeholders preferences for municipal solid waste management plans, Boston, USA. Extraído el 27 de junio de 2012 de http://www.sciencedirect.com/science/article/pii/S09 21344908000372

- Cordón, R. (2008). Enseñanza y aprendizaje de procedimientos científicos (contenidos procedimentales) en la educación secundaria obligatoria. Análisis de la situación, dificultades y perspectivas. (Tesis doctoral en Investigación e Innovación en la Enseñanza de las Ciencias). Murcia: Universidad de Murcia, Departamento de Didáctica de las Ciencias Experimentales.

- Corral Q., S. y Quintero, M. E. (2007). La metodología multicriterial y los métodos de valoración de impactos ambientales. Extraído el 28 de agosto de 2012 de http://www.saber.ula.ve/bitstream/123456789/17402/ 1/articulo4.pdf

- Chen, S., Jiang, Y., Lui, Y. \& Diao, C. (2012). Cost constrained mediation model for analytic hierarchy process negotiated decision making. Journal of MultiCriteria Decision Analysis, 19, 3-13.

- Dalalah, D., Hayajneh, M. \& Batieha, F. (2011). A fuzzy multi-criteria decision making model for supplier selection. Extraído el 06 de agosto de 2012 de http://www.sciencedirect.com/science/article/pii/S09 57417411000510

- Dooley, A. E., Smeaton, D. C., Sheath, G. W. \& Ledgard S. F. (2009). Application of multiple criteria decision analysis in the New Zealand agricultural industry. Extraído el 18 de octubre de 2012 de http://onlinelibrary.wiley.com/doi/10.1002/mcda.437/ full

- Dou, Y., Zhu, L. \& Simon, H. W. (2012). Solving the fuzzy shortest path problem using multi-criteria decision method based on vague similarity measure. Extraído el 17 de junio de 2012 de 
http://www.sciencedirect.com/science/article/pii/S15 68494612001019

- Duglio, I. (2007). Los prácticos de laboratorio: una mirada interpretativa en prácticas de enseñanza de química en bachillerato diversificado. Universidad ORT Uruguay. Instituto de Investigación. Cuadernos de Investigación Educativa, Vol. 2. No. 14. Uruguay.

- Font, G. (2000). Gestión de la información en la utilización del proceso analítico jerárquico para la toma de decisiones de nuevos productos. Extraído el 13 de agosto de 2011 de http://redalyc.uaemex.mx/redalyc/src/inicio/ArtPdfR ed.jsp?iCve $=63500304$

- Funtowicz, S. y Ravetz, J. R. (1993). Epistemología política. Ciencia con la gente. Extraído el 15 de febrero de 2011 de http://www.coodi.com.uy/redoeste/docs/menu\%20g eneral/MATERIALES\%20DE\%20INTERES/Epistemol ogia\%20politica\%20Ciencia\%20con\%20

- Furio B., E. (2005). Los lenguajes de la Economía. Edición digital a texto completo accesible en www.eumed.net/libros/2005/efb/

- Garmendia, E. \& Gamboa, G. (2012). Weighting social preferences in participatory multi-criteria evaluations: $A$ case study on sustainable natural resource management. Extraído el 15 de febrero de 2011 de http://ideas.repec.org/p/bcc/wpaper/2012-06.html

- Gómez-Limón, J. A. y Berbel, J. (2000). Multicriteria analysis of derived water demand functions: a Spanish case study. Extraído el 08 de noviembre de 2012 de http://www.sciencedirect.com/science/article/pii/S03 08521X9900075X

- Gómez López, R. (2004). Evolución científica y metodológica de la economía: Escuelas de pensamiento. Extraído el 10 de marzo de 2011 de http://www.eumed.net/cursecon/libreria/

- Gómez, R. D., Yepes D., C. E., Rodríguez O., F. L., Roldán J., P., Velásquez V., W., Lopera V., J., Martínez R., A. O., Vargas P., G. S., Agudelo V., N. A. y Agudelo L., S. M. (2009). Manual de gestión de proyectos. Extraído el 10 de mayo de 2011 de http://aprendeenlinea.udea.edu.co/lms/moodle/file.p hp/551/MANUAL_GESTION PROYECTOS.pdf

- González, A. y Garza, R. (2003). Aplicación de las técnicas multicriteriales en la evaluación y selección de proveedores. Extraído el 20 de noviembre de 2012 de http://rii.cujae.edu.cu/index.php/revistaind/article/vi ewArticle/189

- Hajkowicz, S. (2007). A comparison of multiple criteria analysis and unaided approaches to environmental decision making. Extraído el 20 de noviembre de 2012 de

http://www.sciencedirect.com/science/article/pii/S14 6290110600116X

- Hajkowicz, S. \& Higgins, A. (2008). A comparison of multiple criteria analysis techniques for water resource 
management. Extraído el 20 de noviembre de 2012 de http://www.sciencedirect.com/science/article/pii/S03 77221706011039

- Hernández, R., Fernández, C. y Baptista, P. (2006). Metodología de la Investigación. 5 Ed. McGraw Hill, México. 497p. ISBN 968-422-931-3.

- Lakatos, I. (2007). La metodología de los programas de investigación científica. 2 ed. Madrid: Alianza.

- León R., Nohra. (2006). Elementos para la valoración de pasivos ambientales en hidrocarburos para Colombia. Extraído el 14 de marzo de 2011 de http://fich.unl.edu.ar/CISDAV/upload/Ponencias_y_P osters/Eje06/

Nohra_Leon_Rodriguez/Le\%C3\%B3n_Elementos\%2 Opara\%20la\%20

valoraci\%C3\%B3n\%20de\%20pasivos\%20ambientale s.pdf

- Linkov, I., Satterstrom, F. K., Kiker, G., Batchelor, C., Bridges, T. \& Ferguson, E. (2006). From comparative risk assessment to multi-criteria decision analysis and adaptive management: Recent developments and applications. Extraído el 25 de septiembre de 2012 de http://www.lisdmmp.org/MeetingMaterials/Resource s/EnvIntl_1485.pdf

- Mendoza, G. A. \& Martins, H. (2006). Multi-criteria decision analysis in natural resource management: $A$ critical review of methods and new modelling paradigms. Extraído el 21 de septiembre de 2012 de http://www.sciencedirect.com/science/article/pii/S03 78112706002258

- McDowell, L. (2000). Género, identidad y lugar. Extraído el 10 de marzo de 2011 de http://books.google.com.co/books?id=yTjBaj6HGlg C\&pg=PA365\&lpg $=P A 365 \& d q=J .+A l l e n+26+L .+$ Mcdowell \&source $=b \mid \&$ ots=P9g5FJlpRg\&sig =2cGZTeaAibf3Uju4Td8TjnFQCr4\&hl=es\&sa=X\&ei= P683UJiqEqO36QHKmo

DwCA\&ved=0CDIQ6AEwAQ\#v=onepage \&q=J.\%20AI len $\% 20 \% 26 \% 20$ L. $\% 20 M c d o w e l l \& f=$ false

- Montibeller, G. \& Franco, A. (2010). Multi-Criteria decision analysis for strategic decision making. En: Zopounidis, C. \& Pardalos, P. M. (eds.), Handbook of multicriteria analysis (pp. 25-48). Heidelberg: Verlag Berlin Heidelber.

- Moselhi, O. \& Roofigari-Esfahan, N. (2012). Compression of Project Schedules using the Analytical Hierarchy Process. Extraído el 2 de noviembre de 2012 de http://onlinelibrary.wiley.com/doi/10.1002/mcda.490/ full

- Munda, G. (1993). Información difusa en los modelos de evaluación multicriterio Ambiental. (Tesis de Doctorado). Ámsterdam: Universidad Libre de Amsterdam.

- (2004). Social multi-criteria evaluation: Methodological foundations and operational consequences. Extraído el 31 de octubre de 2012 de 
http://www.sciencedirect.com/science/article/pii/S03 77221703003692

- Munda, G., Nijkamp, P. \& Rietveld, P. (1995). Qualitative multicriteria methods for fuzzy evaluation problems: An illustration of economic-ecological evaluation. Extraído el 31 de octubre de 2012 de http://www.sciencedirect.com/science/article/pii/037 7221793E02502

- Ortegón, E., Pacheco, J. F. y Roura, H. (2005). Metodología general de identificación, preparación y evaluación de proyectos de inversión pública. Santiago de Chile: Instituto Latinoamericano y del Caribe de Planificación Económica y Social (ILPES).

- Pacheco, J. F. y Contreras, E. (2008). Manual metodológico de evaluación multicriterio para programas y proyectos. Santiago de Chile: Instituto Latinoamericano y del Caribe de Planificación Económica y Social (ILPES).

- Pedone, C. (2000). El trabajo de campo y los métodos cualitativos. Necesidad de nuevas reflexiones desde las geografías latinoamericanas. Scripta Nova. Revista Electrónica de Geografía y Ciencias Sociales, 57.

- Philip, L. J. (1998). Combining quantitative and qualitative approaches to social research in human geography -an impossible mixture? Extraído el 1 de noviembre de 2012 http://www.envplan.com/abstract.cgi?id=a300261

- Philo, C. (1998). Más palabras, más mundos: reflexiones en torno al 'giro cultural' y a la geografía social. Extraído el 17 de agosto de 2011 de http://ddd.uab.es/pub/dag/02121573n34p81.pdf

- Rodríguez-Becerra, M. y Espinoza, G. (2002). Gestión ambiental en América Latina y el Caribe: Evolución, tendencias y principales prácticas. Extraído el 23 de agosto de 2011 de http://www.manuelrodriguezbecerra.org/bajar/gestio n/capitulo11.pdf

- Rodríguez, D. y Valldeoriola, J. (2009). Metodología de la investigación. Extraído el 11 de octubre de 2011 de http://zanadoria.com/syllabi/m1019/mat_castnodef/PID_00148556-1.pdf

- Roura, H. y Cepeda, H. (1999). Manual de identificación, formulación y evaluación de proyectos de desarrollo rural. Extraído el 12 de julio de 2011 de http://www.eclac.org/publicaciones/xml/9/6729/man ual2.pdf

- Salo, A. \& Hamalainen, R. P. (2010). Multicriteria decision analysis in group decision processes. En: Kilgour, D. M. \& Eden, C. (eds.). Handbook of group decision and negotiation (pp. 269-300). Dordrech.

- Sainz Z., R. O., Álvarez V., R y Henríquez A., F. (2012). Objetivos múltiples para la evaluación del desempeño de centros de investigación. Extraído el 7 de mayo de 2011 de http:/lwww.concyteg.gob.mx/ideasConcyteg/Archiv os/86_5_SAINZ_ET_AL_1.pdf 
- Sayer, A. (1984). Method in Social Science: A realist approach. Extraído el 7 de febrero de 2011 de http://books.google.com.co/books? id=q31fv7rFujgC \&pg=PA296\&lpg

$=P A 296 \& d q=A L .+$ Sayer $+\% 26+K .+$ Morgan $\&$ source $=b \mid$ \&ots=b2XZ_gWBUg\&sig=zeNkPsV7XIUI90CK8M07JVwBCM\&hl=es\#v=onepage\&q $=$ AL.\%20Sayer $\% 20 \% 26 \% 20 \mathrm{~K} . \% 20$ Morgan \& $\mathrm{f}=$ false

- Simões-Gomes, C. F., Nunes, K. R. A., Xavier, L. H., Cardoso, R. \& Valleb, R. (2008). Multicriteria decision making applied to waste recycling in brazil. Extraído el 7 de febrero de 2011 de http://www.sciencedirect.com/science/article/pii/S03 $0504830600123 X$

- Stirling, A. (2006). Analysis, participation and power: justification and closure in participatory multi-criteria analysis. Land use policy, 23, 95-107.

- Toskano H., G. B. (2005). El Proceso de análisis jerárquico como herramienta para la toma de decisiones en la selección de proveedores. Monografía. Facultad de Ciencias Matematicas. E.A.P. de Investigacion Operativa. Universidad Nacional Mayor de San Marcos. Lima, Perú.

- Uribe T., D. A. (2001). La evaluación multicriterio y su aporte en la construcción de una función de valor económico total para los bosques en piedras blancas. (Tesis de Maestría en Bosques y Conservación Ambiental). Medellín: Universidad Nacional de Colombia sede Medellín, Facultad de Ciencias Agropecuarias.

- Vargas, O. L. (2005). La evaluación multicriterio social y su aporte a la conservación de los bosques. Extraído el 1 de febrero de 2012 de http://www.scielo.org.co/scielo.php?pid=S0304-

28472 005000100007\&script=sci_arttext

- Vizcaíno, J., Picuasi, M. y Quiroz, M. C. (2011). Deficiencia en el desarrollo de la motricidad fina en niños y niñas de 4 a 5 años de la "Unidad Educativa Sagrado Corazón de Jesús de la Ciudad de Ibarra". Propuesta alternativa. Extraído el 1 de octubre de 2012 de http://hdl.handle.net/123456789/397

- Wen-Hsiang, W., Chang-tzu, Ch. \& Chin-tsai L. (2008). Comparing the aggregation methods in the analytic hierarchy process when uniform distribution. Extraído el 1 de noviembre de 2012 de http://www.wseas.us/elibrary/transactions/economics/2008/Bus-Eco.pdf

- Winchester, H. (1996). Ethical issues in interviewing as a research method in human geography. Extraído el 9 de octubre de 2012 de http://www.tandfonline.com/doi/abs/10.1080/000491 89608703161\#preview

- Zerguini, S., Khademi, N., Mohaymany, A. S. \& Shahi J. (2011). An Algorithm for the Analytic Network Process (ANP) Structure Design. Extraído el 17 de septiembre 
de

2012

de

http://halshs.archives-ouvertes.fr/hal-00696339/

1. Ph.D. en Ciencias Agrarias. Profesor Asociado, Departamento de Producción Agropecuaria, Universidad de Caldas. alberto.grajales@ucaldas.edu.co

2. Ph.D en Ciencias Económicas. Profesor Titular Departamento de Economía y Administración, Universidad de Caldas. edgar.serrano@ucaldas.edu.co

3. Especialista en Evaluación Técnica y Económica de Impactos Ambientales. Profesor Asociado, Departamento de Producción Agropecuaria, Universidad de Caldas. christine.hahn@ucaldas.edu.co

4. Ciencia como "saber" podemos definirla en general como "[...] un modo de conocimiento que aspira a formular, mediante lenguajes rigurosos y apropiados, en lo posible con el auxilio del lenguaje matemático, leyes por medio de las cuales se rigen los fenómenos. Estas leyes son de diversos órdenes. Todas tienen varios elementos en común: ser capaces de describir series de fenómenos, ser comprobables por medio de la observación de los hechos y ser capaces de predecir acontecimientos futuros" (Ferrater, 1965 citado por Gómez, 2004, p. 10).

5. Una variable cuantitativa es aquella variable que representa una característica o propiedad del objeto de estudio que puede cuantificarse y puede ser expresada con números. Una variable cualitativa es una variable que representa una propiedad que hace referencia a cualidades del objeto de estudio y no puede ser expresada cuantitativamente. 